\title{
Free Vibration of a Perfectly Clamped-Free Beam with Stepwise Eccentric Distributed Masses
}

\author{
Gilbert-Rainer Gillich,, Zeno-Iosif Praisach,, Magd Abdel Wahab, ${ }^{2}$ Nicoleta Gillich, \\ Ion Cornel Mituletu, ${ }^{1}$ and Codrin Nitescu ${ }^{1,3}$ \\ ${ }^{1}$ Department of Mechanical Engineering, Faculty of Engineering and Management, "Eftimie Murgu" University of Resita, \\ P-ta Traian Vuia 1-4, 320085 Resita, Romania \\ ${ }^{2}$ Soete Laboratory, Faculty of Engineering and Architecture, Ghent University, Technologiepark Zwijnaarde 903, \\ 9052 Zwijnaarde, Belgium \\ ${ }^{3} B M W$ AG, Hufelandstrasse 4, 80937 München, Germany
}

Correspondence should be addressed to Gilbert-Rainer Gillich; gr.gillich@uem.ro

Received 18 October 2015; Revised 18 January 2016; Accepted 19 January 2016

Academic Editor: Tai Thai

Copyright ( $) 2016$ Gilbert-Rainer Gillich et al. This is an open access article distributed under the Creative Commons Attribution License, which permits unrestricted use, distribution, and reproduction in any medium, provided the original work is properly cited.

A direct approach for the calculation of the natural frequencies and vibration mode shapes of a perfectly clamped-free beam with additional stepwise eccentric distributed masses is developed, along with its corresponding equations. Firstly there is contrived influence of a mass, located on a given position along the beam, upon the modal energies, via an energy analysis method. Secondly, the mass participation coefficient is defined as a function of the mass location and the bending vibration mode number. The proposed coefficient is employed to deduce the mathematical relation for the frequencies of beams with supplementary eccentric loads, generally available for any boundary conditions. The accuracy of the obtained mathematical relation was examined in comparison with the numerical simulation and experimental results for a cantilever beam. For this aim, several finite element models have been developed, individualized by the disturbance extent and the mass increase or decrease. Also, one real system was tested. The comparisons between the analytically achieved results and those obtained from experiments proved the accuracy of the developed mathematical relation.

\section{Introduction}

During operation, engineering structures are subjected to loads, often produced by supplementary masses. These structures can suffer various damage types, including corrosion due to the interaction with the environment, which produce alterations of the geometry and the mechanical/physical properties [1]. All these alterations from the original state affect the structure's modal parameters. Some typical damage geometries are known. Among cracks, one can distinguish between breathing cracks, producing just a stiffness decrease, and open cracks, which additionally generate a decrease in mass. Regarding corrosion, a conventional criterion to classify this phenomenon is concerned with the appearance of the corroded area. The two basic forms of corrosion are defined as (a) generalized corrosion, when the components surface is affected at the same rate on a large area, and (b) localized corrosion, which is restricted to compact areas [2].

Development of damage detection methods has drawn the interest of numerous researchers [3-8]. Even if most papers presented in literature tackle open cracks, the influence of the loss of mass is neglected, introducing this way inaccuracy in the damage detection methods. This fact is also valid for the detection of corrosion in structures. Another aspect concerns the design of damage detection methods for structures loaded by supplementary masses. Thus, deriving simple mathematical relations, which are able to predict the frequency changes due to mass alteration, is an actual challenge.

Herein, the cases of mass increase and decrease are equally treated, for different beam segment lengths, affected by these mass distortions. The idea was to find a reliable 


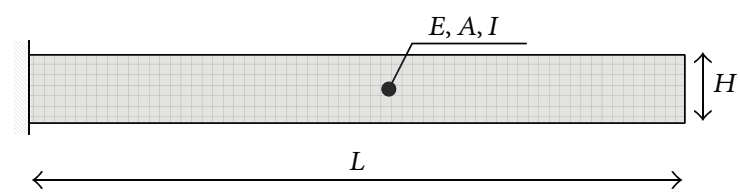

FIgURE 1: Cantilever beam model.

equation, which is able to indicate the natural frequencies, valid for beams irrespective of their support type and for any mass change scenario.

In prior research devoted to the analysis of beams with open cracks (see $[9,10]$ ), slim beam regions were considered affected by stiffness and mass changes. It was proved that the superposition principle can be applied to find the natural frequencies of beams with open cracks. As a consequence, the problem of stiffness and mass variation can be approached separately. This paper introduces a behavioral model and the subsequent mathematical relation for beams with uniform stiffness but variable distributed mass, for which the accuracy is proved by comparison with numerical simulations and experimental test results.

\section{Analytical Investigation}

The aim of this section is to introduce a mathematical relation contrived by the authors, which predicts the frequency changes due to mass variation. In order to illustrate the case, a cantilever beam is used. In this example, the geometrical asymmetry assures an unequivocal link between the mass disturbance, defined by position and intensity, and the frequency changes. The analyzed prismatic beam of steel, illustrated in Figure 1 , has length $L$, width $B$, and thickness $H$. As a consequence, the beam has a cross-sectional area $A=$ $B \cdot H$ and a moment of inertia $I=\left(B \cdot H^{3}\right) / 12$. The mechanical parameters involved in this analysis are the longitudinal elasticity modulus $E$, the volumetric mass density $\rho$, and Poisson's ratio $\mu$. In addition, the earth gravity $g$ is considered. On the cantilever beam only the dead mass is considered.

The natural frequency $f_{i}$ of the $i$ th bending vibration mode for a beam with any support type is derived as

$$
f_{i}=\frac{\lambda_{i}^{2}}{2 \pi} \sqrt{\frac{E I}{m L^{3}}}
$$

where $m$ is the beam mass and $\lambda_{i}$ are characteristic roots achieved as solutions of the characteristic equation representing four boundary conditions. For the cantilever beam, this equation is

$$
\cos \lambda \cosh \lambda+1=0 .
$$

The beam subjected to a supplementary load, induced by a mass $m_{a}$ uniformly distributed on a segment of length $\Delta L$ located between points $a$ and $b$, is shown in Figure 2(a). The beam's model is depicted in Figure 2(b) in a continuous approach and in Figure 2(c) for the finite element analysis. In this last case, the total mass growth is achieved by increasing the volumetric mass density of the mentioned segment (marked with dark color). On the contrary, if the segment between points $a$ and $b$ has a reduced mass, a diminished volumetric mass density in the region is taken into account. The beam stiffness is kept unchanged in all cases.

For finding the influence of mass changes upon the beam dynamics, the cantilever beam is considered as continuous and without mass. A mass $m_{E}$, positioned at the free end, covers in a quarter-period $T_{i} / 4$ the distance $w_{i}(L)$. Another mass $m_{P}$, located at the distance $x$ from the fixed end of the beam, covers at the same time the distance $w_{i}(x)$. These distances are different for various vibration modes and depend on the bending mode shapes. For the cantilever beam, these are described as

$$
\begin{aligned}
w_{i}(x)= & \cosh \lambda_{i} x-\cos \lambda_{i} x \\
& -\frac{\cos \lambda_{i} L+\cosh \lambda_{i} L}{\sin \lambda_{i} L+\sinh \lambda_{i} L}\left(\sinh \lambda_{i} x-\sin \lambda_{i} x\right) .
\end{aligned}
$$

Figure 3(a) illustrates the cases of a mass located at the free end, while Figure 3(b) depicts one at an intermediate position, both for the bending vibration mode two.

The kinetic energy calculated for the tip mass $m_{E}$ is

$$
U_{K i}(L)=\frac{m_{E}}{2}\left[\frac{w_{i}(L)}{T_{i}}\right]^{2} .
$$

For the mass $m_{P}$ located at the distance $x$, the general case, the kinetic energy is given by

$$
U_{K i}(x)=\frac{m_{P}}{2}\left[\frac{w_{i}(x)}{T_{i}}\right]^{2} .
$$

If the kinetic energies in the two cases are equal, the same natural frequency for them both is obtained. The dependency between the two masses $m_{E}$ and $m_{P}$ for the $i$ th vibration mode results from (4) and (5), as

$$
m_{E i}=m_{P}\left[\frac{w_{i}(x)}{w_{i}(L)}\right]^{2}=m_{P}\left[\bar{w}_{i}(x)\right]^{2} .
$$

Note that, for the cantilever beam, the largest displacements are always achieved at the free end. Thus, $\bar{w}_{i}(x)$ is achieved by normalization and denotes the dimensionless transverse displacement for a point placed at the distance $x$ from the fixed end. Obviously, $\left[\bar{w}_{i}(x)\right]^{2}$ takes values between zero and one. In the case of cantilever beams, (6) shows that the tip mass has the most important contribution to the total kinetic energy. Also, (6) indicates that any mass located at a distance $x$ from the fixed end can be replaced by an equivalent mass located at the free end. The scaling factor employed to find the equivalent mass is given by the square of the normalized mode shape $\left[\bar{w}_{i}(x)\right]^{2}$. From the dynamic point of view, the beam's equivalent mass is achieved by summarizing the equivalent masses of all beam slices. In Figure 4, this fact is illustrated for the case of the second bending vibration mode. Actually, the total equivalent beam mass $\bar{m}_{i \text {-eq }}$ placed at the free end, corresponding to the $i$ th bending vibration mode, is obtained by multiplying the specific mass $\bar{m}$ with the area under the curve $\left[\bar{w}_{i}(x)\right]^{2}$. 


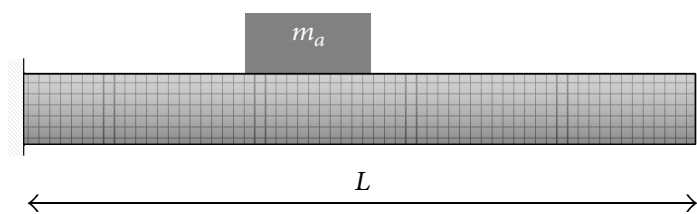

(a)

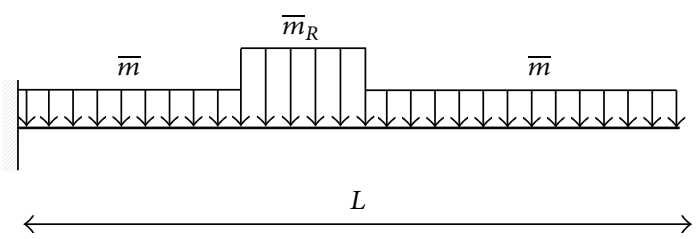

(b)

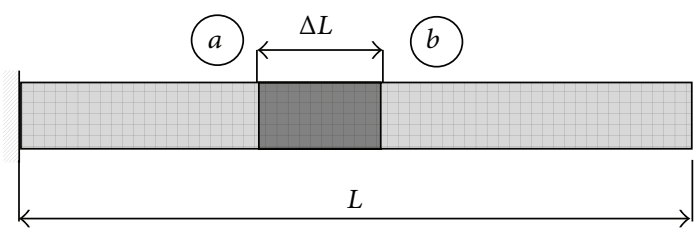

(c)

Figure 2: Cantilever beam with additional mass and its continuous and finite element models.

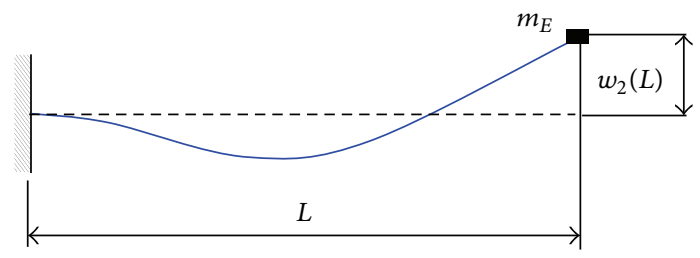

(a)

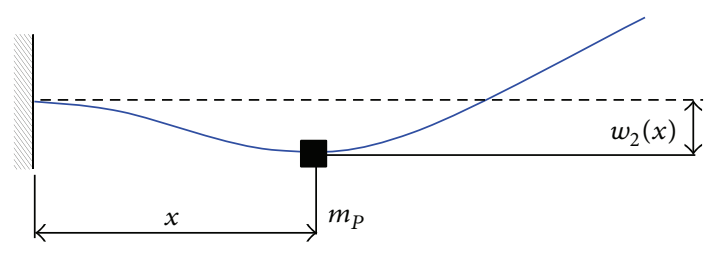

(b)

FIGURE 3: Dynamically equivalent systems having the mass located at different positions.

Consequently, the total equivalent mass, for the ith vibration mode, is

$$
m_{i-\mathrm{eq}}=\bar{m} L \int_{0}^{L}\left[\bar{w}_{i}(x)\right]^{2} d x=\varsigma_{i}^{0-L} \bar{m} L=0.25 \bar{m} L
$$

The term $\varsigma_{i}^{0-L}=\int_{0}^{L}\left[\bar{w}_{i}(x)\right]^{2} d x$ was nominated as the mass participation coefficient. The superscript of $\varsigma_{i}^{0-L}$ stands for the considered segment limits, in this case being the whole beam length. It is worth underlining that for all vibration modes the area under the curve $\left[\bar{w}_{i}(x)\right]^{2}$ in the interval limits $0-L$ is 0.25 , irrespective of the vibration mode.

Therefore, the frequency can be expressed with regard to the equivalent mass, as

$$
f_{i}=\frac{\lambda_{i}^{2}}{2 \pi} \sqrt{\frac{E I}{\bar{m} L^{4}}}=\frac{\lambda_{i}^{2}}{2 \pi} \sqrt{\frac{E I}{4 m_{i-\mathrm{e} L} L^{3}}} .
$$

The contribution to the total kinetic energy can be derived from (7) as the sum of the mass participation of all infinitesimal slices. The individual mass participation considers the distance covered in a quarter of the period $T_{i}$, so that it is different for the various vibration modes. Figure 5 indicates the normalized mass participation of 100 slices for the second bending vibration mode.

Now, the beam with a uniform cross section loaded with supplementary mass on a beam segment of length $\Delta L$ placed between points $a$ and $b$ was considered. The load was applied in a manner which did not affect the beam stiffness. This
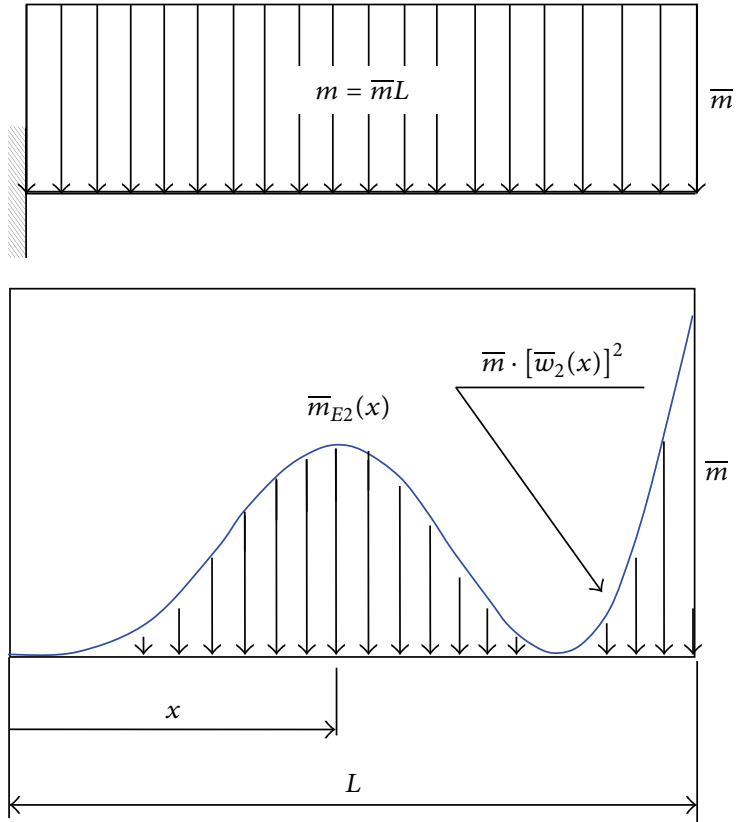

FIGURE 4: The uniformly distributed inertial mass, loading on the cantilever beam in mode two, and the mass participation in the kinetic energy of each slice.

can be modeled by an increased volumetric mass density, resulting in an increased specific mass $\bar{m}_{R}$ on that segment. 


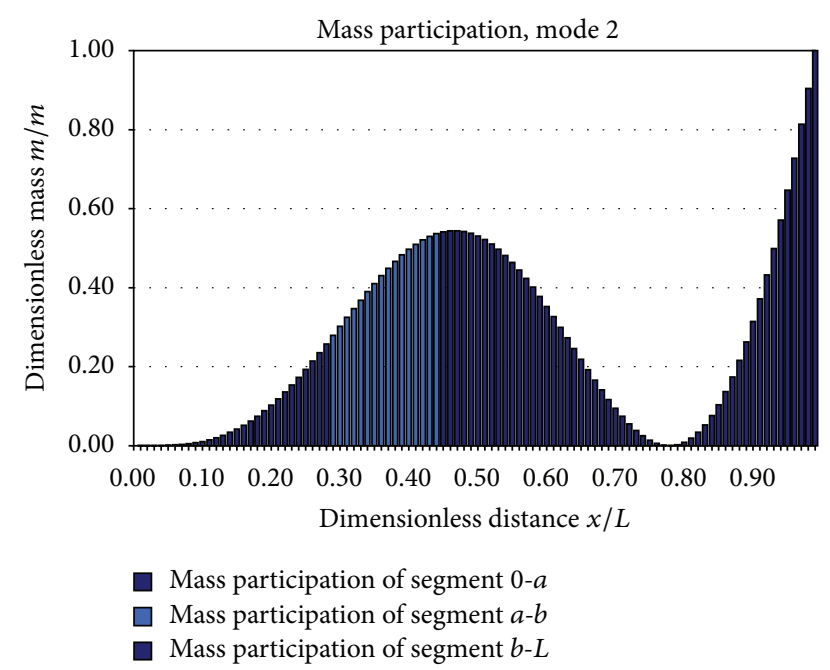

FIGURE 5: The mass participation in the total kinetic energy of the uniformly distributed loads for 100 slices placed along the beam.

The equivalent mass contribution of the segment subjected to a mass increase is

$$
m_{R i-\mathrm{eq}}^{a-b}=\bar{m}_{R} L \int_{a}^{b}\left[\bar{w}_{i}(x)\right]^{2} d x=\varsigma_{i}^{a-b} \bar{m}_{R} L .
$$

The total equivalent specific mass, contributing to the kinetic energy, is found by summarizing the equivalent specific masses of this segment and the other two segments, which are not subjected to any additional mass. These last two are

$$
\begin{aligned}
& m_{i-\mathrm{eq}}^{0-a}=\bar{m} L \int_{0}^{a}\left[\bar{w}_{i}(x)\right]^{2} d x=\varsigma_{i}^{0-a} \bar{m} L, \\
& m_{i-\mathrm{eq}}^{b-L}=\bar{m} L \int_{b}^{L}\left[\bar{w}_{i}(x)\right]^{2} d x=\varsigma_{i}^{b-L} \bar{m} L,
\end{aligned}
$$

where $\varsigma_{i}^{0-a}, \varsigma_{i}^{a-b}$, and $\varsigma_{i}^{a-L}$ are the mass participation coefficients for segments $0-a, a-b$, and $b-L$, respectively.

Figure 6 depicts the mass participation for a beam having an increased mass density on a segment with the limits $a=0.3 \mathrm{~L}$ and $b=0.45 \mathrm{~L}$. On that segment, the increased distributed mass has as effect the drop of the kinetic energy. For the beam subjected to an additional mass, the entire mass contribution is presented in Figure 6(a). One can observe the distortion of the mass participation coefficients between points $a$ and $b$.

In the case of a supposed uniform stiffness, the natural frequencies for the beam with increased mass between points $a$ and $b$, that is, $\bar{m}_{R}>\bar{m}$, are

$$
f_{R i}=\frac{\lambda_{i}^{2}}{2 \pi} \sqrt{\frac{E I}{\left(m_{i-\mathrm{eq}}^{0-a}+m_{R i-\mathrm{eq}}^{a-b}+m_{i-\mathrm{eq}}^{b-L}\right) L^{3}}}
$$

or, considering the mass participation coefficients,

$$
f_{R i}=\frac{\lambda_{i}^{2}}{2 \pi} \sqrt{\frac{E I}{\left(\varsigma_{i}^{0-a} \bar{m}+\varsigma_{i}^{a-b} \bar{m}_{R}+\varsigma_{i}^{b-L} \bar{m}\right) L^{4}} .}
$$

Obviously, the natural frequencies decrease due to an additional mass. In the case of mass loss, that is, $\bar{m}_{R}<\bar{m}$, the frequencies of the beam can be also calculated with (12). In this case, increased natural frequencies are expected.

From (8) and (12), the natural frequencies of the beam with altered mass with respect to the frequencies of the uniform beam are obtained as

$$
f_{R i}=f_{i} \sqrt{\frac{\varsigma_{i}^{0-L} \bar{m}}{\varsigma_{i}^{0-a} \bar{m}+\varsigma_{i}^{a-b} \bar{m}_{R}+\varsigma_{i}^{b-L} \bar{m}}}
$$

If the beam is subjected to the action of more masses, the frequencies can be similarly derived, but a particular approach is imposed for each specific segment. This means that in (13) more terms will be present in the denominator.

Equation (13) can be employed also for the case of inertial loads which are not uniformly distributed along segment $a-b$. In this case $m_{R i \text {-eq }}=c_{i}^{a-b} f\left(\bar{m}_{R}\right)$ and is expressed with respect to the mass variation along the $a$-b beam segment $f\left(\bar{m}_{R}\right)$. In this regard, it is possible to derive the effect of a variable cross section upon the frequencies of such a beam. Additionally, if the stiffness variation is known, it is possible to find out the beam frequencies for variable cross section, by superposing the effects of stiffness and mass alteration.

In practical applications, such as damage detection or vibration control, the frequency deviation caused by the mass alteration is relevant. It is

$$
\Delta f_{R i}=f_{i}\left(\sqrt{\frac{\varsigma_{i}^{0-L} \bar{m}}{\varsigma_{i}^{0-a} \bar{m}+\zeta_{i}^{a-b} \bar{m}_{R}+\zeta_{i}^{b-L} \bar{m}}}-1\right) .
$$

From (14), it clearly results in the fact that by increasing the mass the frequency changes reaching negative values, whereas, by a loss of mass, the change is in the sense of frequency increase.

The relative frequency shift, achieved by dividing the frequency change due to mass alteration to that of the uniform beam, becomes

$$
\Delta \tilde{f}_{R i}=\frac{\Delta f_{R i}}{f_{i}}=\sqrt{\frac{\varsigma_{i}^{0-L} \bar{m}}{\left(\varsigma_{i}^{0-a} \bar{m}+\varsigma_{i}^{a-b} \bar{m}_{R}+\varsigma_{i}^{b-L} \bar{m}\right)}}-1 .
$$

Because the contrived relations, expressing the frequency of the nonuniformly loaded beam, the frequency shift, and the relative frequency shift of transverse modes, contain just terms dependable on the support type (e.g., mode shapes and wave numbers), the relations are generally valid. The single requirement is the involvement of these features obtained for the right boundary conditions. In the next sections, the obtained mathematical relations are examined as opposed to numerical simulations and experimental results in the case of a cantilever beam.

\section{Numerical Verification}

To prove the correctness of the contrived mathematical relations, by means of the finite element analysis (using 


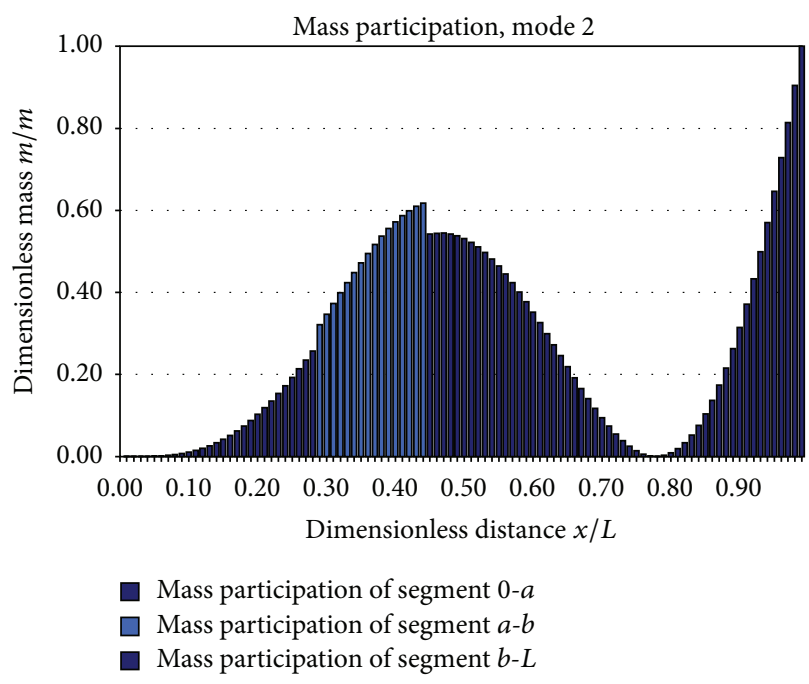

(a)

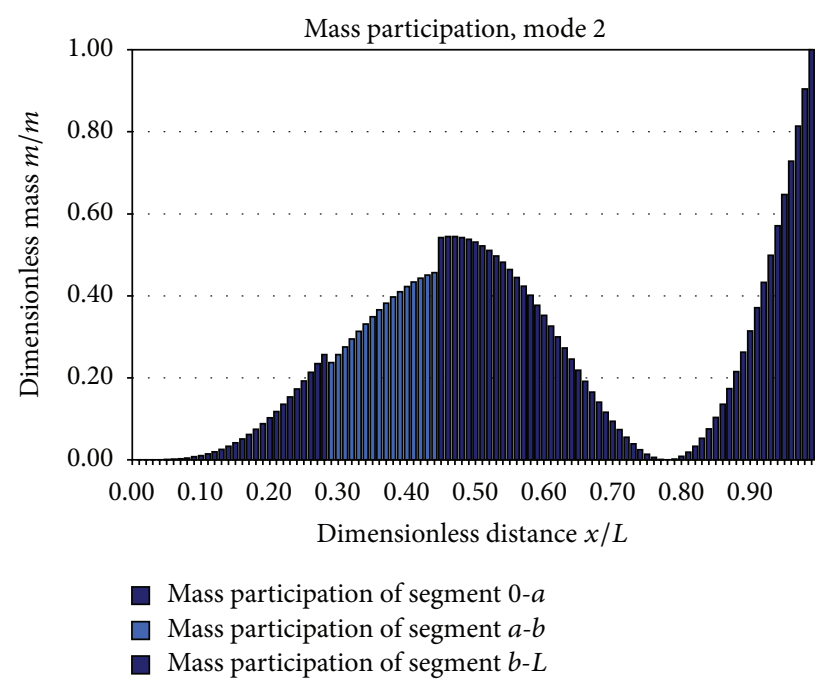

(b)

FIGURE 6: The mass participation in the total kinetic energy for 100 slices placed along the beam: (a) in case of supplementary mass; (b) in case of mass loss.

TABLE 1: Parameters reflecting the segments affected by mass alteration, large regions.

\begin{tabular}{lcccc}
\hline Scenario & Mass density $\rho\left[\mathrm{kg} / \mathrm{m}^{3}\right]$ & Left limit $a[\mathrm{~mm}]$ & Right limit $b[\mathrm{~mm}]$ & Extent $\Delta L[\mathrm{~mm}]$ \\
\hline A & 4000 & 300 & 400 & 100 \\
B & 10000 & 300 & 600 & 100 \\
C & 12000 & 600 & 750 & 50 \\
D & 2000 & 700 & 750 & 50 \\
E & 14000 & 500 & 550 & 50 \\
F & 3000 & 800 & 850 & 50 \\
\hline
\end{tabular}

ANSYS software), a series of simulations were performed. The initial investigations have taken into consideration the uniform beam, having a length $L=1 \mathrm{~m}$ and a rectangular cross section with the following geometry: width $B=50 \mathrm{~mm}$ and thickness $H=5 \mathrm{~mm}$. The physical-mechanical properties are the volumetric mass density $\rho=7850 \mathrm{~kg} / \mathrm{m}^{3}$, the longitudinal elasticity modulus $E=2.0 \cdot 10^{11} \mathrm{~N} / \mathrm{m}^{2}$, and the Poisson ratio $\mu=0.3$. The gravitational acceleration is taken to $g=9.806 \mathrm{~m} / \mathrm{s}^{2}$, the mass of the beam becoming $m=$ $1.9625 \mathrm{~kg}$. The first three natural frequencies of the transverse modes are derived, in order to get the reference values, which are presented in Table 2.

Afterwards, beams presenting nonuniform mass distributions were considered. The particular cases are individualized by the disturbance position, extent, and intensity. Regarding the disturbance extent, two typical cases are defined: (a) deviation from the uniform mass distribution present on large segments and (b) slim regions affected by the altered mass load. It has to be noted that the mass increase or decrease is modeled by a changed mass density of the respective segment. The parameters of the regions subjected to mass alteration are comprehensively presented in Tables 1 and 2 .

The first three natural frequencies for the transverse vibration modes are found using the modal analysis module. Tables 3 and 4 present the results derived for the uniform beam using (1) and for the twelve designed scenarios by involving the contrived equation (13). The results achieved by simulations are presented for comparison in Tables 5 and 6. The fit between the results analytically obtained and by means of FEA confirms the validity of the derived equations.

The relative frequency shifts are derived and compared to have a better overview on the results. Tables 7 and 8 show the results achieved by analytical study and FEA.

Taking a look at Tables 7 and 8, a good fit between the relative frequency shifts derived analytically and by means of FEA simulation is observed. This good concordance is highlighted in Figures 7-10, where the comparison is separately made for the same class of mass alterations (regarding the extension and the sense of mass change). Thus, the correctness of the mathematical relations presented in Section 2 is confirmed.

The relative frequency shifts (RFSh) have got a special attention. They clearly characterize any nonuniformity in the beam's mass distribution, indicating at the same time the location and level of supplementary load. For the same position, the RFSh differ by amplitudes, which are in a relation defined by the additional load level (e.g., ratio between the regular density and the density achieved in the region, where the distortion is present). Thus, by normalizing the relative frequency shifts a sequence of values is attained, constituting a pattern that indicates the region in which the mass 
TABLE 2: Parameters reflecting the segments affected by mass alteration, slim regions.

\begin{tabular}{lcccc}
\hline Scenario & Mass density $\rho\left[\mathrm{kg} / \mathrm{m}^{3}\right]$ & Left limit $a[\mathrm{~mm}]$ & Right limit $b[\mathrm{~mm}]$ & Extent $\Delta L[\mathrm{~mm}]$ \\
\hline $\mathrm{G}$ & 4000 & 100 & 110 & 10 \\
$\mathrm{H}$ & 10000 & 100 & 10 & 10 \\
$\mathrm{I}$ & 12000 & 150 & 160 & 10 \\
$\mathrm{~J}$ & 2000 & 150 & 210 & 10 \\
$\mathrm{~K}$ & 14000 & 200 & 210 & 10 \\
$\mathrm{~L}$ & 3000 & 200 & 10 \\
\hline
\end{tabular}

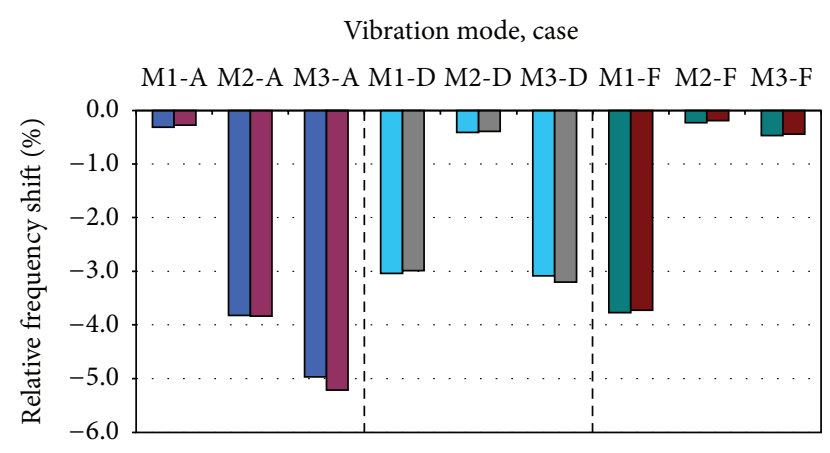

FIGURE 7: The relative frequency shifts analytically derived (left bars for each mode) and evaluated by FEA (bars positioned at right) for the mass increase on an extended region.

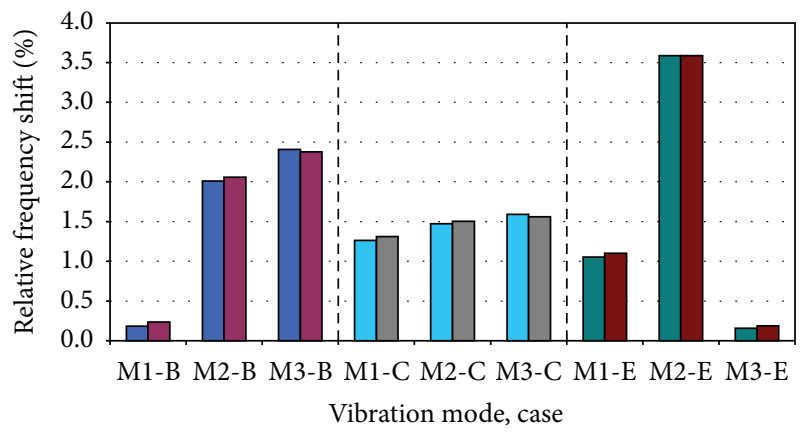

FIGURE 8: The relative frequency shifts analytically derived (left bars for each mode) and evaluated by FEA (bars positioned at right) for the mass decrease on an extended region.

Vibration mode, case

M1-G M2-G M3-G M1-J M2-J M3-J M1-L M2-L M3-L

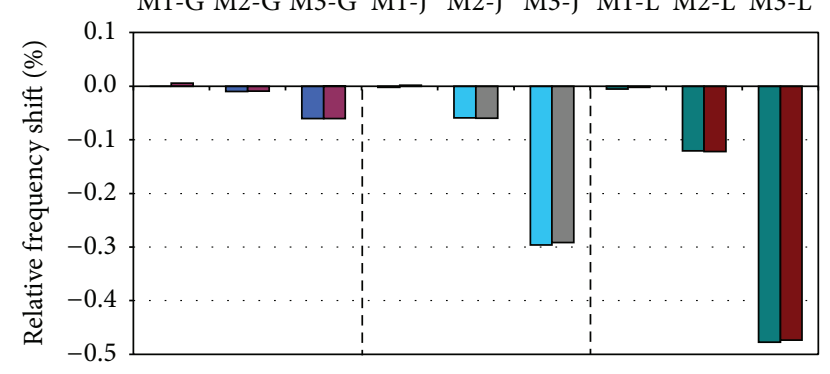

FIGURE 9: The relative frequency shifts analytically derived (left bars for each mode) and evaluated by FEA (bars positioned at right) for the mass increase on a narrow segment.
TABLE 3: Natural frequencies analytically derived for wide affected region scenarios.

\begin{tabular}{cccc}
\hline \multirow{2}{*}{ Mode $i$} & Uniform beam & \multicolumn{2}{c}{ Beam with stepped load } \\
& $f_{i}[\mathrm{~Hz}]$ & Scenario & $f_{R i}[\mathrm{~Hz}]$ \\
\hline \multirow{3}{*}{1} & A & 4.08977 \\
& & B & 4.06976 \\
& & C & 4.02542 \\
& D & 4.20082 \\
& & E & 4.03397 \\
& F & 4.23059 \\
\hline \multirow{3}{*}{25.54951} & A & 26.52667 \\
& B & 25.04867 \\
& C & 25.17304 \\
& D & 25.65486 \\
& & E & 24.63272 \\
& & F & 25.60767 \\
\hline \multirow{3}{*}{3} & A & 75.09158 \\
& & B & 69.76202 \\
& & C & 70.40146 \\
& & D & 73.74612 \\
& & E & 71.42629 \\
& & F & 71.87511 \\
\hline
\end{tabular}

nonuniformity is present. These patterns are in direct relation to the square of the mode shape value at the affected region location and they can be analytically defined if the boundary conditions are known.

\section{Laboratory Experiments}

The experimental tests have been designed to complete the numerical analysis, in order to prove the correctness of the theoretical findings. These were carried out on a cantilever beam similar to that chosen for the FEA. Here, the first seven natural frequencies of the weak axis bending modes, for the uniform beam and the beam subjected to an additional mass (see scenario E), are targeted. To assure the clamped end with a proper fixing, the beam was clamped in a vise, as presented in Figure 11.

The acquisition system consists of a laptop, a compact chassis (NI cDAQ-9172) with NI 9234 signal acquisition modules, and a Kistler 8772 accelerometer that is fixed close to the beam's free end. Since a high precision in evaluating 
TABLE 4: Natural frequencies analytically derived for concentrated mass loads.

\begin{tabular}{cccc}
\hline \multirow{2}{*}{ Mode $i$} & Uniform beam & \multicolumn{2}{c}{ Beam with stepped load } \\
& $f_{i}[\mathrm{~Hz}]$ & Scenario & $f_{R i}[\mathrm{~Hz}]$ \\
\hline \multirow{3}{*}{4} & $\mathrm{G}$ & 4.07691 \\
& & $\mathrm{H}$ & 4.07689 \\
& & $\mathrm{I}$ & 4.07683 \\
& & $\mathrm{~J}$ & 4.07699 \\
& & $\mathrm{~K}$ & 4.07661 \\
& $\mathrm{~L}$ & 4.07713 \\
\hline \multirow{3}{*}{25.54951} & $\mathrm{G}$ & 25.55209 \\
& & $\mathrm{H}$ & 25.54805 \\
& $\mathrm{I}$ & 25.53872 \\
& $\mathrm{~J}$ & 25.56472 \\
& & $\mathrm{~K}$ & 25.51040 \\
& & $\mathrm{~L}$ & 25.58046 \\
\hline \multirow{3}{*}{3} & $\mathrm{G}$ & 71.58216 \\
& & $\mathrm{H}$ & 71.51493 \\
& & $\mathrm{I}$ & 71.39000 \\
& & $\mathrm{~J}$ & 71.75063 \\
& & $\mathrm{~K}$ & 71.11268 \\
& & $\mathrm{~L}$ & 71.88067 \\
\hline
\end{tabular}

TABLE 5: Natural frequencies found by FEA for large affected region scenarios.

\begin{tabular}{|c|c|c|c|}
\hline \multirow{2}{*}{ Mode $i$} & \multirow{2}{*}{$\begin{array}{c}\text { Uniform beam } \\
f_{i}^{\mathrm{FEM}}[\mathrm{Hz}]\end{array}$} & \multicolumn{2}{|c|}{ Beam with stepped load } \\
\hline & & Scenario & $f_{R i}^{\mathrm{FEM}}[\mathrm{Hz}]$ \\
\hline \multirow{6}{*}{1} & \multirow{6}{*}{4.08986} & A & 4.10330 \\
\hline & & B & 4.08236 \\
\hline & & $\mathrm{C}$ & 4.03839 \\
\hline & & $\mathrm{D}$ & 4.21423 \\
\hline & & $\mathrm{E}$ & 4.04695 \\
\hline & & $\mathrm{F}$ & 4.24427 \\
\hline \multirow{6}{*}{2} & \multirow{6}{*}{25.62655} & A & 26.62145 \\
\hline & & B & 25.11124 \\
\hline & & $\mathrm{C}$ & 25.25335 \\
\hline & & $\mathrm{D}$ & 25.73864 \\
\hline & & $\mathrm{E}$ & 24.718923 \\
\hline & & $\mathrm{F}$ & 25.68827 \\
\hline \multirow{5}{*}{3} & \multirow{5}{*}{71.75447} & $\mathrm{~A}$ & 75.53598 \\
\hline & & B & 70.08363 \\
\hline & & $\mathrm{C}$ & 70.67144 \\
\hline & & $\mathrm{D}$ & 74.09155 \\
\hline & & $\mathrm{E}$ & 71.65492 \\
\hline
\end{tabular}

the natural frequencies is required, due to the expected small frequency changes, the implementation of a properly developed virtual instrument in the LabVIEW environment was necessary [11]. It allows the acquisition of the acceleration time history and an accurate evaluation of frequencies [12,13].

A noncontact acoustic excitation was employed to produce controlled input. The acoustic excitation system chain
TABLE 6: Natural frequencies found by FEA for concentrated mass loads.

\begin{tabular}{cccc}
\hline \multirow{2}{*}{ Mode $i$} & $\begin{array}{c}\text { Uniform beam } \\
f_{i}^{\text {FEM }}[\mathrm{Hz}]\end{array}$ & \multicolumn{2}{c}{ Beam with stepped load } \\
& & Scenario & $f_{R i}^{\text {FEM }}[\mathrm{Hz}]$ \\
\hline \multirow{4}{*}{4} & $\mathrm{G}$ & 4.08963 \\
& & $\mathrm{H}$ & 4.08963 \\
& & $\mathrm{I}$ & 4.08961 \\
& & $\mathrm{~J}$ & 4.08979 \\
& $\mathrm{~K}$ & 4.08947 \\
& & $\mathrm{~L}$ & 4.08993 \\
\hline \multirow{3}{*}{25.62655} & $\mathrm{G}$ & 25.62894 \\
& & $\mathrm{H}$ & 25.62506 \\
& $\mathrm{I}$ & 25.61647 \\
& $\mathrm{~J}$ & 25.64190 \\
& & $\mathrm{~K}$ & 25.58856 \\
& & $\mathrm{~L}$ & 25.65777 \\
\hline \multirow{3}{*}{3} & $\mathrm{G}$ & 71.79798 \\
& & $\mathrm{H}$ & 71.73294 \\
& & $\mathrm{I}$ & 71.60898 \\
& & $\mathrm{~J}$ & 71.96386 \\
& & $\mathrm{~K}$ & 71.32889 \\
& & $\mathrm{~L}$ & 72.09396 \\
\hline
\end{tabular}

TABLE 7: The relative frequency shifts for large affected region scenarios.

\begin{tabular}{cccc}
\hline Mode $i$ & Scenario & $\Delta \widetilde{f}_{R i}[\%]$ & $\Delta \widetilde{f}_{R i}^{\text {FEM }}[\%]$ \\
\hline \multirow{4}{*}{1} & A & -0.3156 & -0.2763 \\
& B & 0.1843 & 0.2353 \\
& C & 1.2625 & 1.3101 \\
& D & -3.0397 & -2.9871 \\
& E & 1.0527 & 1.1008 \\
& F & -3.769 & -3.7212 \\
\hline \multirow{4}{*}{3} & A & -3.8246 & -3.8318 \\
& B & 2.0091 & 2.0584 \\
& C & 1.4734 & 1.5041 \\
& D & -0.4124 & -0.3886 \\
& E & 3.5882 & 3.5885 \\
F & -0.2277 & -0.1921 \\
\hline & A & -4.9659 & -5.2150 \\
& B & 2.4087 & 2.3796 \\
& C & 1.5901 & 1.5608 \\
& D & -3.0852 & -3.2030 \\
& E & 0.1575 & 0.1909 \\
& F & -0.4698 & -0.4443 \\
\hline
\end{tabular}

consists of virtual free software, acoustic amplifier with frequency equalizer, and low-frequency speaker. The SweepGen free software installed on the laptop allows a large variety of sound wave manipulations. It provides single or band frequency generation with values between 1 and $100 \mathrm{kHz}$, stepor sweep-variation of frequency in different time periods, and high adjusting of the signal amplitude. The acoustic amplifier ensures, by means of the equalizer adjusting, a clean signal 
TABLE 8: The relative frequency shifts for the regions affected by concentrated loads.

\begin{tabular}{|c|c|c|c|}
\hline Mode $i$ & Scenario & $\Delta \tilde{f}_{R i}[\%]$ & $\Delta \widetilde{f}_{R i}^{\mathrm{FEM}}[\%]$ \\
\hline \multirow{6}{*}{1} & G & -0.0003 & 0.0055 \\
\hline & $\mathrm{H}$ & 0.0002 & 0.0054 \\
\hline & I & 0.0016 & 0.0060 \\
\hline & $\mathrm{J}$ & -0.0023 & 0.0016 \\
\hline & $\mathrm{K}$ & 0.0070 & 0.0095 \\
\hline & $\mathrm{L}$ & -0.0056 & -0.0019 \\
\hline \multirow{6}{*}{2} & G & -0.0101 & -0.0093 \\
\hline & $\mathrm{H}$ & 0.0057 & 0.0058 \\
\hline & I & 0.0422 & 0.0393 \\
\hline & $\mathrm{J}$ & -0.0596 & -0.059 \\
\hline & $\mathrm{K}$ & 0.1530 & 0.1482 \\
\hline & $\mathrm{L}$ & -0.1212 & -0.1218 \\
\hline \multirow{6}{*}{3} & G & -0.0603 & -0.0606 \\
\hline & $\mathrm{H}$ & 0.0336 & 0.0299 \\
\hline & I & 0.2083 & 0.2027 \\
\hline & $\mathrm{J}$ & -0.2958 & -0.2918 \\
\hline & $\mathrm{K}$ & 0.5959 & 0.5931 \\
\hline & $\mathrm{L}$ & -0.4776 & -0.4731 \\
\hline
\end{tabular}

TABLE 9: Natural frequencies of the uniform beam.

\begin{tabular}{lccc}
\hline Mode $i$ & $f_{i}[\mathrm{~Hz}]$ & $f_{i}^{\mathrm{FEM}}[\mathrm{Hz}]$ & $f_{i}^{\mathrm{M}}[\mathrm{Hz}]$ \\
\hline 1 & 4.07690 & 4.08986 & 4.045 \\
2 & 25.54951 & 25.62655 & 25.956 \\
3 & 71.53939 & 71.75447 & 72.654 \\
4 & 140.18865 & 140.62745 & 142.615 \\
5 & 231.74189 & 232.52003 & 235.801 \\
6 & 346.18225 & 347.45183 & 352.505 \\
7 & 483.51075 & 485.45783 & 491.164 \\
\hline
\end{tabular}

TABLE 10: Natural frequencies of the beam loaded by a stepped mass (scenario E).

\begin{tabular}{lccc}
\hline Mode $i$ & $f_{R i}[\mathrm{~Hz}]$ & $f_{R i}^{\mathrm{FEM}}[\mathrm{Hz}]$ & $f_{R i}^{\mathrm{M}}[\mathrm{Hz}]$ \\
\hline 1 & 4.03397 & 4.046953 & 3.988 \\
2 & 24.63272 & 24.71892 & 25.044 \\
3 & 71.42629 & 71.65493 & 72.528 \\
4 & 135.42063 & 136.1956 & 138.036 \\
5 & 230.28665 & 231.3189 & 234.551 \\
6 & 335.93814 & 338.4368 & 343.192 \\
7 & 477.90911 & 480.9907 & 485.748 \\
\hline
\end{tabular}

wave up to $100 \mathrm{~W}$ output power, impedance of $4 \mathrm{ohms}$, and 2 to $22000 \mathrm{~Hz}$ frequency range. The speaker is also adequate in case of low-frequency, having a free-air resonant frequency of about $65 \mathrm{~Hz}$ and providing good linearity before and after this frequency up to $0.5 \mathrm{~Hz}$ in low and $5 \mathrm{kHz}$ in high.
TABLE 11: Relative frequency shifts.

\begin{tabular}{lccc}
\hline Mode $i$ & $\Delta \tilde{f}_{R i}[\%]$ & $\Delta \tilde{f}_{R i}^{\mathrm{FEM}}[\%]$ & $\Delta \widetilde{f}_{R i}^{\mathrm{M}}[\%]$ \\
\hline 1 & 1.0528 & 1.04913 & 1.409147 \\
2 & 3.5882 & 3.54176 & 3.513638 \\
3 & 0.1575 & 0.13873 & 0.173425 \\
4 & 3.370280 & 3.15148 & 3.210742 \\
5 & 0.597020 & 0.51657 & 0.530108 \\
6 & 2.928860 & 2.59462 & 2.641948 \\
7 & 1.095870 & 0.92019 & 1.102687 \\
\hline
\end{tabular}

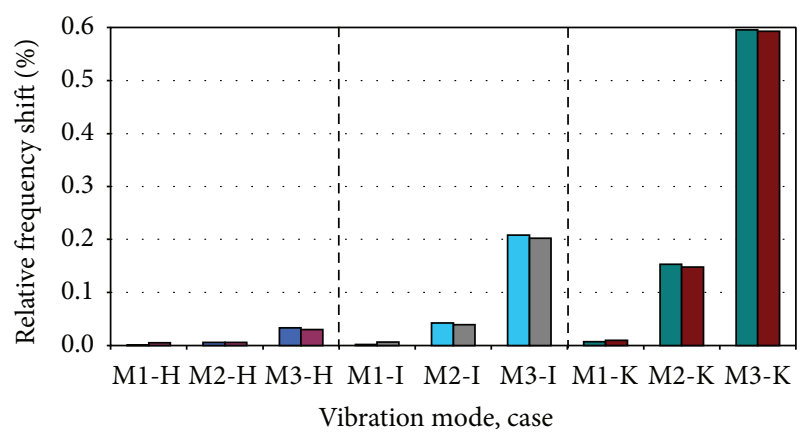

FIGURE 10: The relative frequency shifts analytically derived (left bars for each mode) and evaluated by FEA (bars positioned at right) for the mass decrease on a narrow segment.

Additional masses are distributed between points $a=$ $500 \mathrm{~mm}$ and $b=550 \mathrm{~mm}$ to ensure the same mass load as in scenario $\mathrm{E}$ of the numerical simulations section. Thus, 10 elements, each having the mass around of $7 \mathrm{~g}$ (to attain the additional mass of $76.88 \mathrm{~g}$ ), are distributed along the region of interest. A special care was given to achieve a point contact between the added mass's elements and the beam and to avoid the self-contact between the elements of the added masses, in order to maintain the original stiffness. The way it was adequately performed is detailed in Figure 11(b).

The measured natural frequencies $f_{i}^{\mathrm{M}}$ for the first seven bending vibration modes of the uniform beam as well as that obtained by calculus $f_{i}$ and simulations $f_{i}^{\mathrm{FEM}}$ are presented in Table 9.

Table 10 indicates the frequency values achieved by calculus, FEA, and measurements, for a beam corresponding to scenario E. Taking a look at Tables 9 and 10, one can remark the good fit between the frequencies for the three approaches.

The relative frequency shifts are calculated and compared to accomplish a deeper analysis. For the three approaches, the results are indicated in Table 11 and depicted in Figure 12. Reviewing this figure, the good correlation between the achieved RFSh values can be clearly seen, confirming the validity of the proposed analytical relation for the prediction of frequency shifts due to mass changes.

The results presented in Figures 7-10 and also 12 show that a disturbance from the uniformly distributed mass state is indicated by the relative frequency shift values of the transverse vibration modes. The values of this vector can be used to indicate the location and severity of the disturbance, 


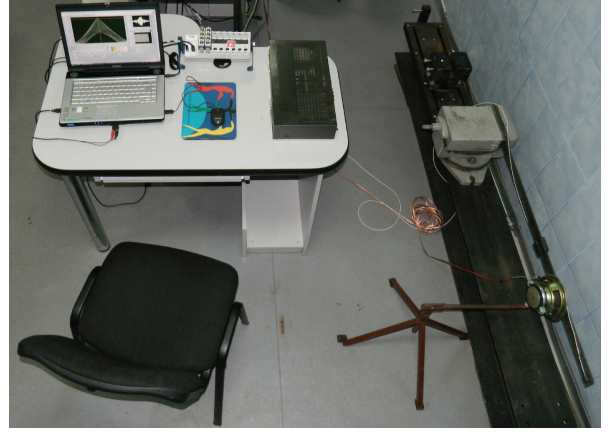

(a)

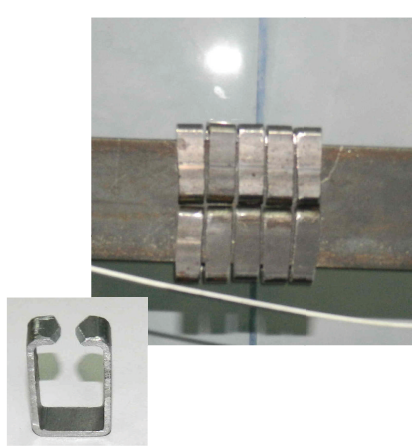

(b)

FIGURE 11: The experimental setup: (a) stand overview; (b) detailed view of the region with added masses.

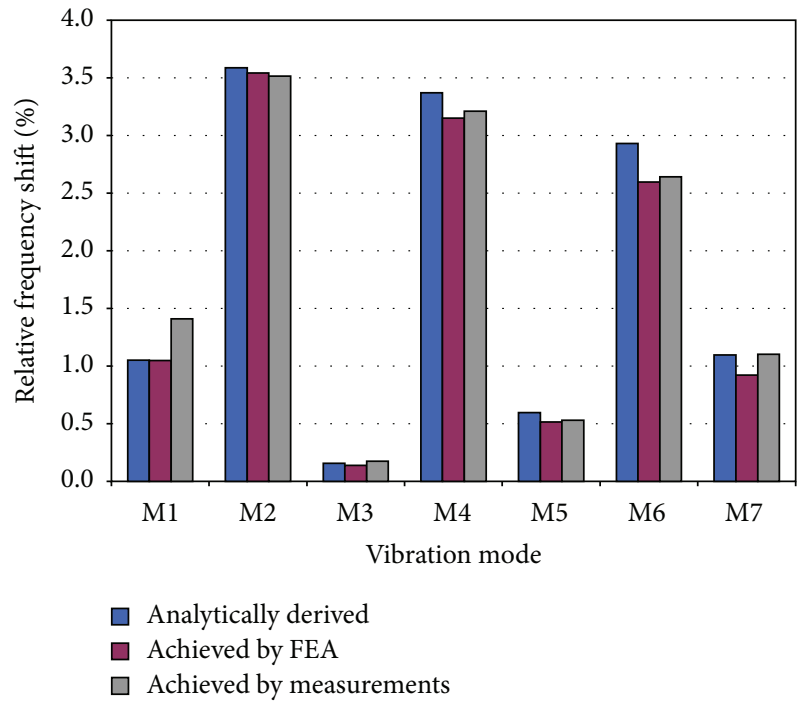

Figure 12: Relative frequency shifts for scenario E.

by involving an inverse method and a pattern recognition algorithm, similar to the case of stiffness decrease [14]. The higher the vibration modes analyzed, the higher the reliability of the results.

\section{Conclusion}

In this paper a mathematical relation for the prediction of frequency changes due to alteration of mass is proposed. This relation associates the effect of a local mass on the natural frequencies with the square of the mode shape corresponding to that location. Since the relation has as input parameters the position, the mass alteration, and the beam mode shape, it becomes easily adaptable to any kind of boundary conditions. The accuracy of the achieved mathematical relation has been examined by numerical simulations and experiments, in the case of a cantilever beam, and the results confirmed its robustness and reliability.

The relative frequency shifts derived from this relation provide a simple way to describe the vibrational behavior of beams with nonuniform distributed mass. Thus, it is possible to create a database containing numerous scenarios afterwards employable to find the region subjected to mass distortion and derive its significance.

\section{Conflict of Interests}

The authors declare that there is no conflict of interests regarding the publication of this paper.

\section{Acknowledgment}

The work has been funded by the Sectoral Operational Programme Human Resources Development 2007-2013 of the Ministry of European Funds through the Financial Agreement POSDRU/159/1.5/S/132395.

\section{References}

[1] A. Morassi and F. Vestroni, Dynamic Methods for Damage Detection in Structures, vol. 499 of CISM Courses and Lectures, Springer, Vienna, Austria, 2008.

[2] R. Landolfo, L. Cascini, and F. Portioli, "Modeling of metal structure corrosion damage: a state of the art report," Sustainability, vol. 2, no. 7, pp. 2163-2175, 2010.

[3] S. W. Doebling, C. R. Farrar, M. B. Prime, and D. W. Shevitz, "Damage identification and health monitoring of structural and mechanical systems from changes in their vibration characteristics: a literature review," Report LA 13070-MS, Los Alamos National Laboratory, Los Alamos, NM, USA, 1996.

[4] O. S. Salawu, "Detection of structural damage through changes in frequency: a review," Engineering Structures, vol. 19, no. 9, pp. 718-723, 1997.

[5] Z. Praisach, G. R. Gillich, and D. E. Birdeanu, "Considerations on natural frequency changes in damaged cantilever beams using FEM," in Latest Trends in Engineering Mechanics, Structures, Engineering Geology, pp. 214-219, 2010.

[6] M. R. Kaloop and J. W. Hu, "Stayed-cable bridge damage detection and localization based on accelerometer health monitoring measurements," Shock and Vibration, vol. 2015, Article ID 102680, 11 pages, 2015.

[7] Y.-Z. Song, C. R. Bowen, H. A. Kim et al., "Non-invasive damage detection in beams using marker extraction and wavelets," 
Mechanical Systems and Signal Processing, vol. 49, no. 1-2, pp. 13-23, 2014.

[8] N. M. M. Maia, J. M. M. Silva, E. A. M. Almas, and R. P. C. Sampaio, "Damage detection in structures: from mode shape to frequency response function methods," Mechanical Systems and Signal Processing, vol. 17, no. 3, pp. 489-498, 2003.

[9] G. R. Gillich, Z. I. Praisach, and C. M. Iavornic, "Reliable method to detect and assess damages in beams based on frequency changes," in Proceedings of the ASME International Design Engineering Technical Conference, article DETC201270094, Chicago, Ill, USA, August 2012.

[10] G.-R. Gillich and Z.-I. Praisach, "Damage-patterns-based method to locate discontinuities in beams," in Health Monitoring of Structural and Biological Systems, vol. 8695 of Proceedings of SPIE, pp. 1-8, San Diego, Calif, USA, March 2013.

[11] D. Onchis-Moaca, G.-R. Gillich, and R. Frunza, "Gradually improving the readability of the time-frequency spectra for natural frequency identification in cantilever beams," in Proceedings of the 20th European Signal Processing Conference (EUSIPCO '12), pp. 809-813, Bucharest, Romania, August 2012.

[12] G. R. Gillich, I. C. Mituletu, I. Negru, M. Tufoi, V. Iancu, and F. Muntean, "A method to enhance frequency readability for early damage detection," Journal of Vibration Engineering Technologies, vol. 3, no. 5, pp. 637-652, 2015.

[13] G. R. Gillich, N. M. M. Maia, I. C. Mituletu, Z. I. Praisach, M. Tufoi, and I. Negru, "Early structural damage assessment by using an improved frequency evaluation algorithm," Latin American Journal of Solids and Structures, vol. 12, no. 12, pp. 2311-2329, 2015.

[14] M. I. Friswell, "Damage identification using inverse methods," Philosophical Transactions of the Royal Society A, vol. 365, no. 1851, pp. 393-410, 2007. 


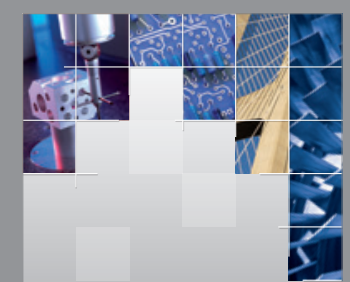

\section{Enfincering}
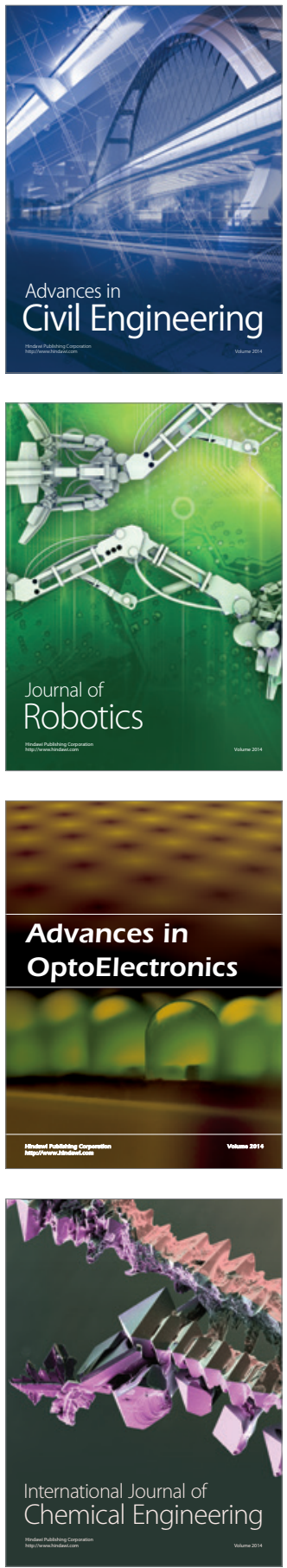

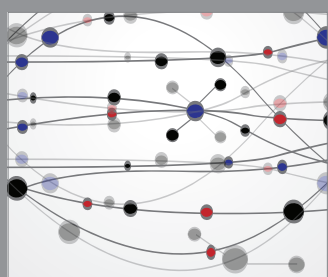

The Scientific World Journal

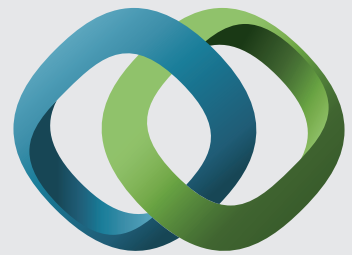

\section{Hindawi}

Submit your manuscripts at

http://www.hindawi.com
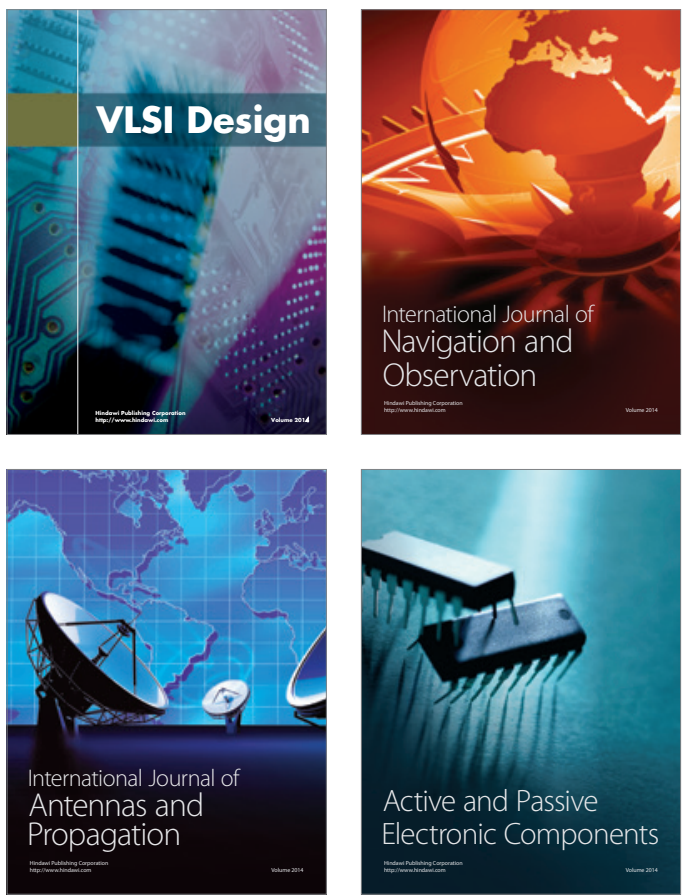
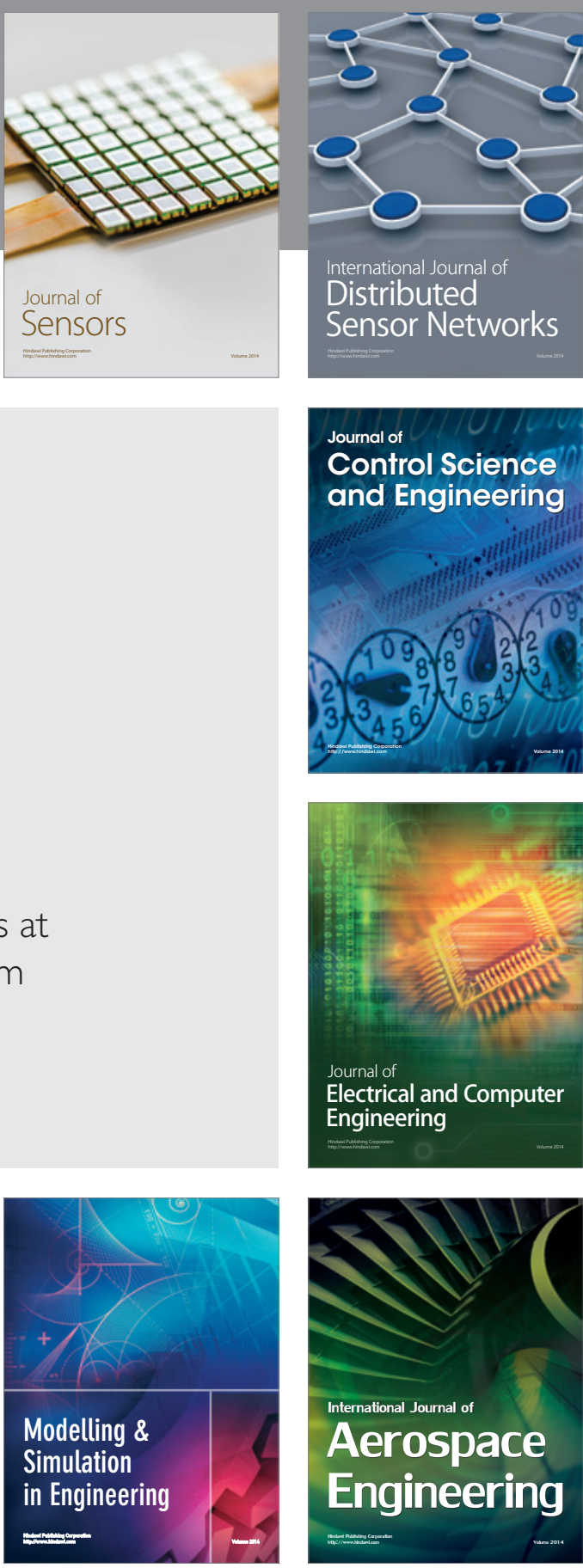

International Journal of

Distributed

Sensor Networks

Journal of

Control Science

and Engineering
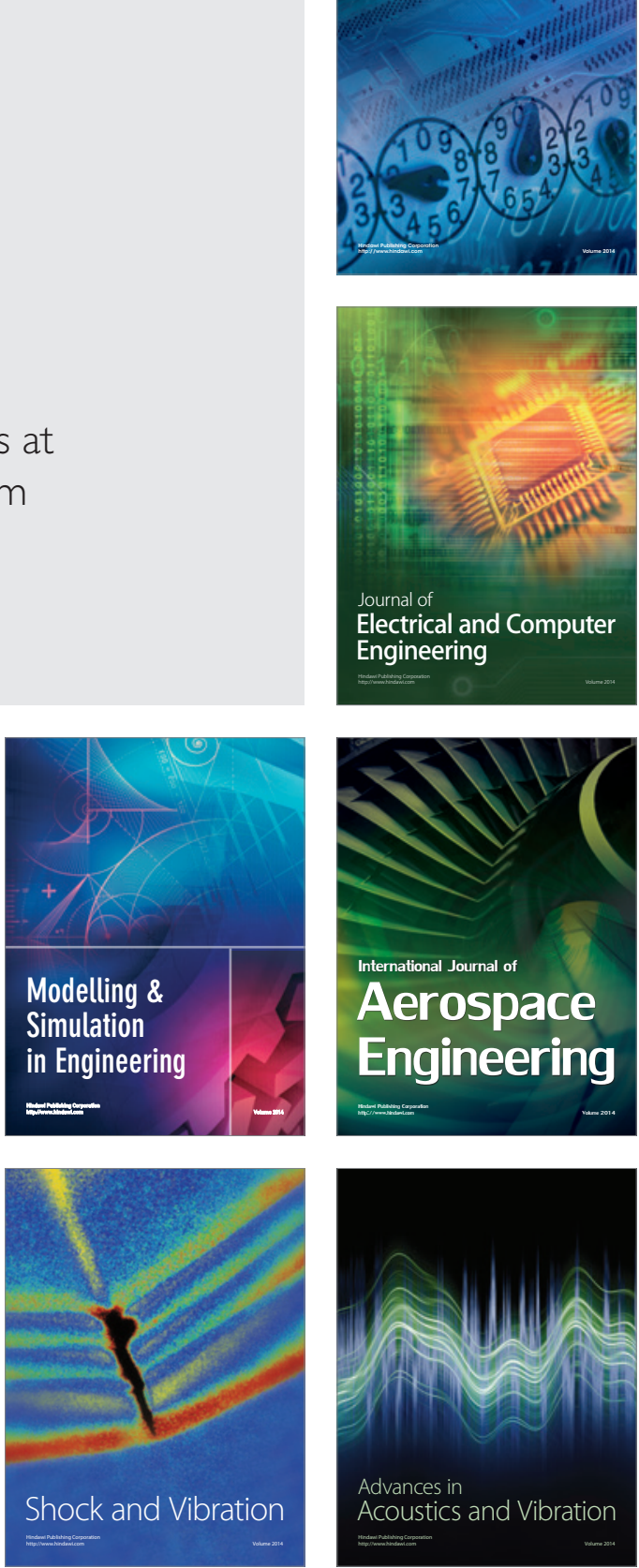